\title{
WHITE MATTER ABNORMALITIES AS ENDOPHENOTYPES OF SCHIZOPHRENIA IN 22q11 DELETION SYNDROME
}

\author{
Marianna Frascarelli, Nikolaos Petsas, Antonino Buzzanca, Martina Fanella, Angelica \\ Maria Monacelli, Tommaso Accinni, Guido Maria Lattanzi, Luca Carlone, Claudio \\ Colonnese, Patrizia Pantano, Massimo Biondi, Fabio Di Fabio
}

Background and aims: 22q11.2 Deletion Syndrome (22q11 DS) patients have $25 \%$ risk of psychosis onset. White matter (WM) brain abnormalities are supposed to be endophenotypes of schizophrenia.

Aim of the present study is to compare WM characteristics, namely Fractional Anisotropy (FA), an indicator of fibers integrity, between groups of schizophrenic and 22q11DS patients to demonstrate they are similar and present also before the psychotic onset.

Methods: Sample consists of 83 subjects, divided into 4 groups: Healthy Controls $(\mathrm{HC}) \mathrm{N}=24 ; 22 q 11 D S$ non-psychotic subjects (DEL) $\mathrm{N}=14$; 22q11DS psychotic patients (DEL_scz) $\mathrm{N}=8$; schizophrenic patients without 22q11DS (SCZ) N=37 (Table 1). 3T Diffusion Tensor Imaging MRI sequences were analysed with Tract Based Spatial Statistic tecnique. Significant results were considered for $p<0.05$ corrected for multiple comparisons.

Results: SCZ and DEL_SCZ groups showed a significant FA reduction compared to $\mathrm{HC}$ in identical clusters of voxels, located in: Corpus Callosum (CC), Fornix, Internal Capsule, Posterior Thalamic Radiation, Inferior and Superior Longitudinal Fasciculi, Inferior Fronto-Occipital Fasciculus, Uncinate Fasciculus.

\begin{tabular}{|c|c|c|c|c|c|c|}
\hline & \multirow{2}{*}{$\begin{array}{c}\mathrm{HC} \\
(\mathrm{N}=24)\end{array}$} & \multirow{2}{*}{$\begin{array}{c}\text { DEL } \\
(\mathrm{N}=14)\end{array}$} & \multirow{2}{*}{$\begin{array}{c}\text { DEL_SCZ } \\
(\mathrm{N}=8)\end{array}$} & \multirow{2}{*}{$\begin{array}{c}S C Z \\
(N=37)\end{array}$} & \multicolumn{2}{|c|}{ STATISTICS } \\
\hline & & & & & $\mathbf{F}$ & \multirow{2}{*}{$\begin{array}{c}\mathbf{p} \\
0,044^{*}\end{array}$} \\
\hline Age (mean $\pm s d)$ & $31,7 \pm 10,8$ & $26,8 \pm 9,3$ & $27,1 \pm 7,4$ & $35 \pm 10,6$ & 2,825 & \\
\hline Gender (\%) & & & & \multicolumn{3}{|c|}{$\chi^{2}$} \\
\hline $\mathbf{F}$ & 58,3 & 28,6 & 25 & 45,9 & \multirow{3}{*}{$\begin{array}{c}4,559 \\
t\end{array}$} & \multirow{3}{*}{0,207} \\
\hline \multirow[t]{2}{*}{ M } & 41,7 & 71,4 & 75 & 54,1 & & \\
\hline & & & CAt & 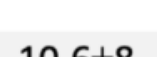 & & \\
\hline (1) & & & 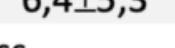 & & & , \\
\hline
\end{tabular}

\section{AVERAGE FA ACROSS THE WHOLE-BRAIN WM SKELETON}

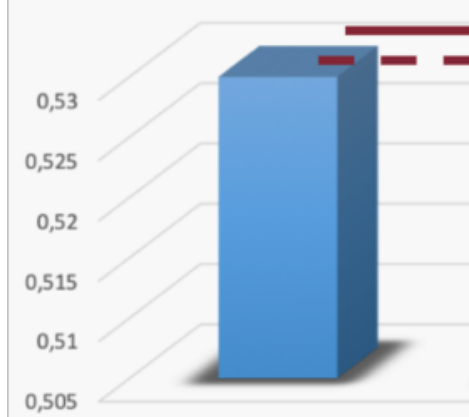

$\mathrm{HC}$

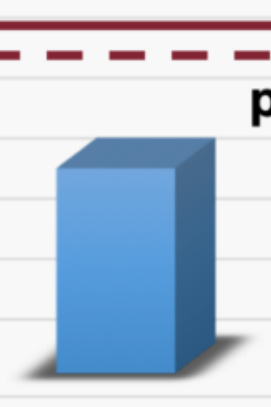

SCZ

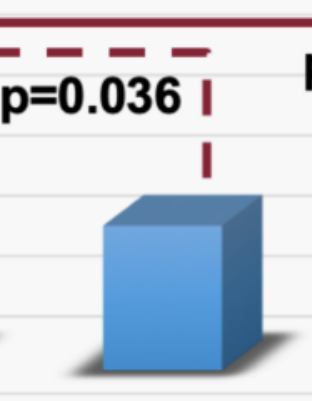

DEL

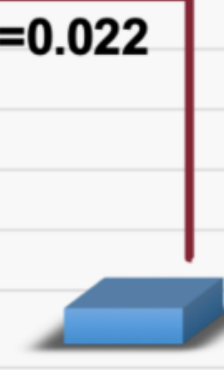

DEL_SCZ
Figure 1. DEL SCZ and DEL showed a global FA reduction comapred to $\mathrm{HC}$. A trend was observed for $\mathrm{SCZ}$ group. WM= white matter.
DEL group showed a significant FA reduction compared to $\mathrm{HC}$ in the same regions (Internal Capsule, Inferior and Superior Longitudinal Fasciculi, Inferior Fronto-Occipital Fasciculus, Fornix and Uncinate Fasciculus) and in the Corona Radiata and External Capsule. The only differences between clinical groups consists of a higher FA in the CC found in DEL, respect to SCZ group.

Conclusions: Whole-brain analyses showed similar reduction of white matter integrity between group at high genetic risk for schizophrenia and patients with an established psychotic disorder. The white matter alterations in schizophrenia seems to be linked to genetic etiopathogenesis of the disorder.

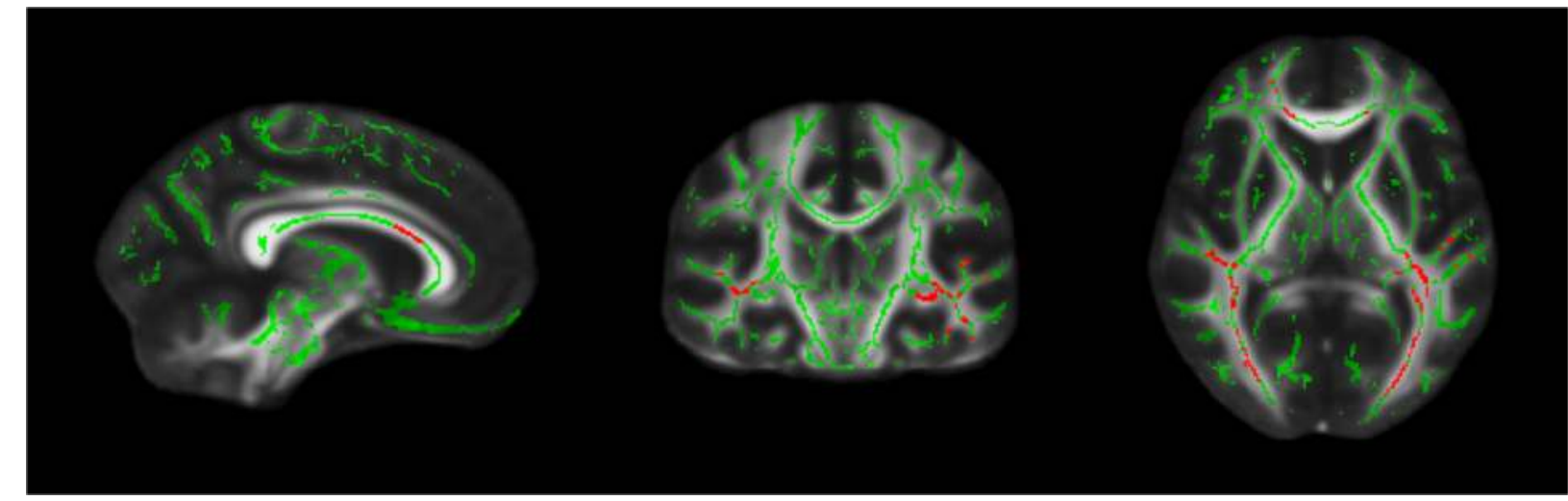

Figure 2. In red FA reduction in SCZ group compared to $\mathrm{HC}$.

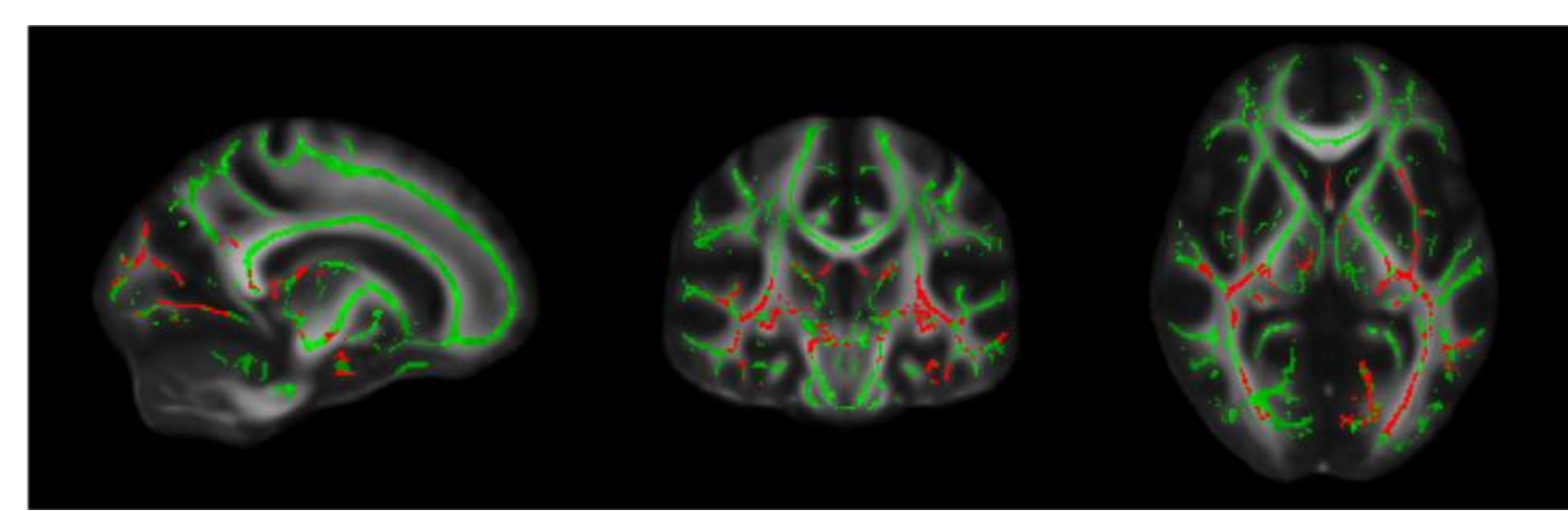

Figure 3. In red FA reduction in DEL_scz group compared to HC.

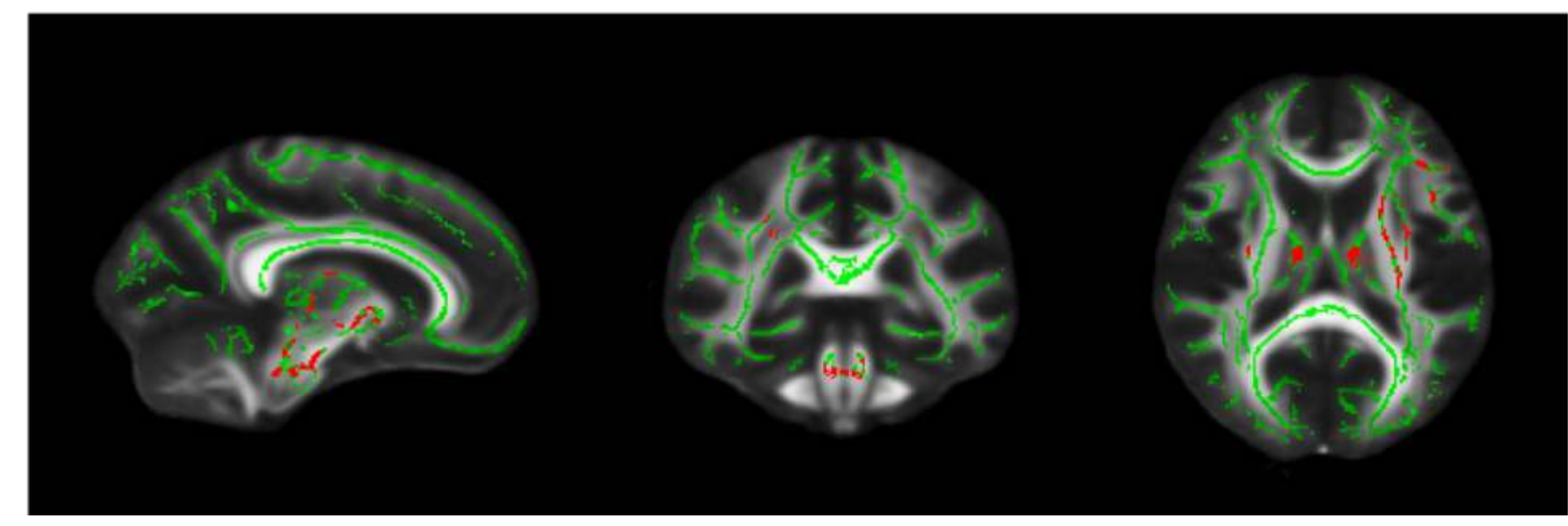

Figure 4. In red FA reduction in DEL group compared to $\mathrm{HC}$.

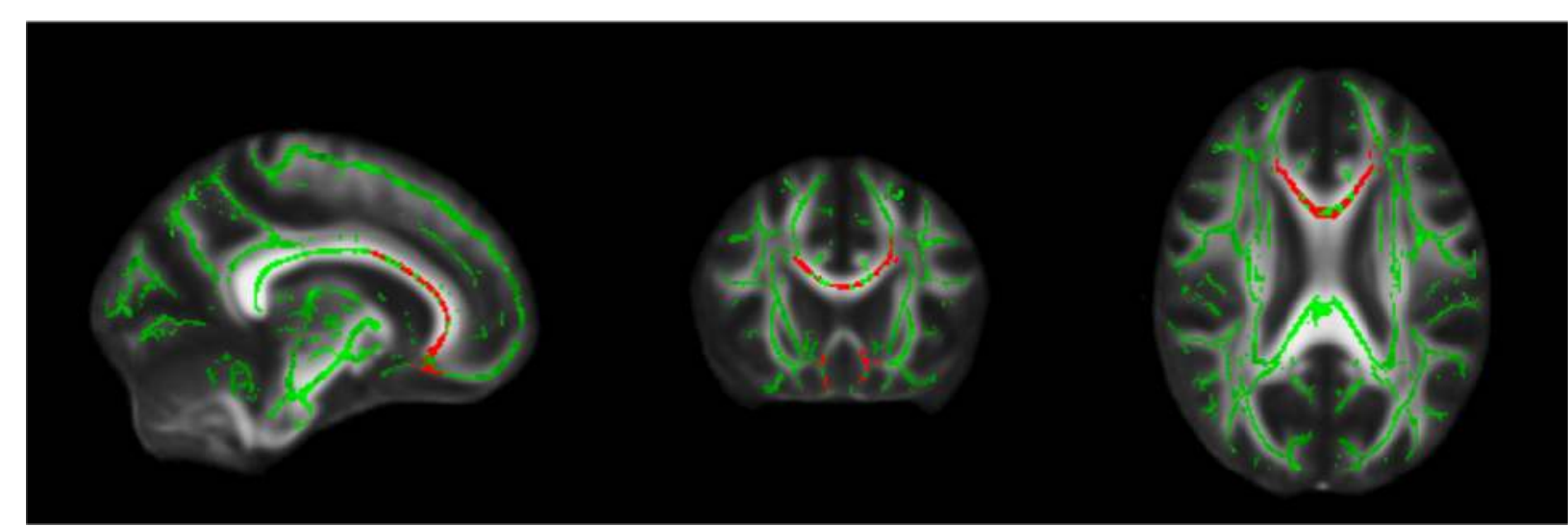

Figure 5. In red FA reduction in SCZ group compared to DEL.

Copyright @ 2017 marianna.frascarelli@gmail.com 